\title{
PREDICTIVE GENES
}

IN BLADDER CANGER

Gene signatures that can predict how patients with bladder cancer will respond to treatment with Bacillus CalmetteGuérin (BCG) have been identified by researchers in South Korea. "Predictive gene signatures can help determine whether patients will benefit from adjuvant BCG treatment or may require more aggressive alternative therapies," says lead researcher Yong-June Kim.

Despite being considered to be the best treatment option for the disease, many patients with nonmuscle-invasive bladder cancer fail to respond to intravesical BCG therapy. Kim and colleagues sought to identify and validate gene signatures that could predict the response to BCG treatment in this patient group.

Microarray gene-expression profiling was carried out in 48 patients with primary pT1 bladder cancer undergoing treatment with BCG immunotherapy to assess the difference in gene expression between responders and nonresponders. 424 genes were significantly associated with recurrence-free survival, and 287 genes were significantly associated with progression-free survival.

Of these gene signatures, 24 genes with the highest expression ratio score were validated in another group of patients with primary PT1 bladder cancer $(n=32)$ treated with BCG immunotherapy. Shorter recurrence or progression times were noted in patients with gene signatures associated with a poor response to treatment with BCG than in those with gene signatures associated with a good response.

Such predictive gene signatures could help to identify patients best suited to treatment with BCG immunotherapy, the authors conclude. "This approach will eventually lead to the development of personalized therapy, thereby significantly lowering the morbidity associated with nonmuscle-invasive bladder cancer," Kim comments.

\section{Lisa Richards}

Original article Kim, Y. J. et al. Gene signatures for the prediction of response to bacillus calmette-guérin immunotherapy in primary pT1 bladder cancers. Clin. Cancer Res. 16, 2131-2137 (2010) 